\title{
Recent developments and present status of telepathology
}

\author{
K. Kayser*, M. Beyer, S. Blum and G. Kayser \\ Department of Pathology, Thoraxklinik, Heidelberg, \\ Germany
}

Telepathology which is the diagnostic work of a pathologist at a distance has been developed to routine application within the last ten years. It can be classified in relation to application, technical solutions, or performance conditions. Diagnostic pathology performance distinguishes primary diagnosis (for example, frozen section statement) from secondary diagnosis (for example, expert consultation) and quality assurance (diagnostic accuracy, continuous education and training). Applications comprise (a) frozen section service; (b) expert consultations; (c) remote control measurements; and (d) education and training. The technical solutions distinguish active (remote control, live imaging) systems from passive (conventional microscope handling, static imaging), and the performance systems with interactive (on-line, live imaging) use from those with passive (offline, static imaging) practice. Intra-operative frozen section service is mainly performed with remote control systems; whereas expert consultations and education/training are commonly based upon Internet connections with static imaging in an off-line mode. The image quality, transfer rates, and screen resolution of active and passive telepathology systems are sufficient for an additional or primary judgment of histological slides and cytological smears. From the technical point of view, remote control telepathology requires a fast transfer and at least near on-line judgement of images, i.e., image acquisition, transfer and presentation can be considered one performance function. Thus, image size, line transfer rate and screen resolution define the practicability of the system. In expert consultation, the pixel resolution of images and natural color presentation are the main factors for diagnostic support, whereas the line transfer rate is of minor importance. These conditions define the technical compartments, especially size and resolution of camera and screen. The performance of commercially available systems has reached a high quality standard. Pathologists can be trained in a short time and use the systems in a routine man-

\footnotetext{
${ }^{*}$ Corresponding author: Prof. Dr. K. Kayser, Department of Pathology, Thoraxklinik, Amalienstr. 5, 69126 Heidelberg, Germany. Tel.: +49 6221396 496; Fax: +49 6221396 238; E-mail: klkayser@ lung.de.
}

ner. Several telepathology systems have been implemented in large Institutes of Pathology which serve for frozen section diagnosis in small hospitals located in the local area. In contrast, expert consultation is mainly performed with international connections. There is a remarkable increase of expert consultations by telepathology according to the experiences of the Armed Forces Institute of Pathology or the Department of Pathology, Thoraxklinik, Heidelberg. In expansion of these experiences, a "globalization" of telepathology can be expected. Telepathology can be used to shrink the period necessary for final diagnosis by request for diagnostic assistance to colleagues working in appropriate related time zones. Telepathology is, therefore, not a substitute of conventional diagnostic procedures but a real improvement in the world of pathology.

Keywords: Telepathology, frozen section service, expert consultation, active telepathology, passive telepathology

\section{Introduction}

The technical progress does not spare the medical disciplines. Progress in medical knowledge is associated with fast split up of so-called conventional medical fields, and improved communication between the different disciplines is required for an optimum use of the detailed knowledge of diseases. In addition, there is need to transfer non-filtered information, i.e., basic information such as images, scattergrams, or curves. Electronic communication in pathology with or without remote robotic components, usually called telepathology has been established in the last years in order to serve for better disease classification and intra-operative services $[5,7,11,12]$. Appropriate equipment is available $[17,20]$. The systems operate most frequently on a point-to-point connection [13, $14,21,24,25,27,31,36,41]$. Most of them provide remote control service [3,24,25,28,31,32,42]. Several reports underlie the practicability and confirm the diagnostic accuracy under various operative conditions [8, $24,26,29,35]$. These systems have been designed for frozen section service; however, the support of intraoperative diagnosis is only one part of telepathology. 
In addition, several terms, such as on-line telepathology, live imaging telepathology, active telepathology, or hybrid systems are used contemporary in articles discussing telepathology $[18,37,42,43]$. Therefore, it seems appropriate to give a survey on these different terms in relation to the practicability, use and performance of telepathology systems. Furthermore, the technical standard of the systems in use, their operation, performance and reported practical experiences will be discussed. These ideas will directly lead to future aspects which can be subsummarized under the term "telepathology globalization". This practice already affects some technical compartments in the daily practice of pathology in some institutions.

\section{Clinical compartments of telepathology}

According to the acknowledged definition of telepathology, which is the diagnostic work of a pathologist at a distance $[15,19]$, the following three medical terms can be distinguished in telepathology: (a) primary telepathology diagnosis, (b) secondary telepathology diagnosis, and (c) quality assurance $[9,15,16]$. The distinction of these three items is important due to the following reasons: Primary diagnosis is performed under time pressure, i.e., the involved pathologist has to provide the clinician with a "crude" diagnosis within a limited time. The time limit is about 10 minutes in intraoperative conditions, and amounts to about 24 hours for biopsy specimens. The "crude" diagnosis has been designed to set up basic therapeutic "directions". These include terms such as malignancy, chronic or acute inflammation, atrophy, etc. Diagnostic details, for example, adenocarcinoma, tuberculosis, etc. are welcome; however, these diagnostic details require frequently additional stains of the specimens which cannot be performed within the time limits of a primary diagnosis. Primary diagnosis is not a "preliminary diagnosis", it only acts on a "crude" level. Secondary diagnosis acts on a more detailed level which depends on the "diagnostic difficulty" and on the disease itself. For example, modern lymphoma diagnosis requires at least immunohistochemistry, and, in addition, often molecular genetic procedures $[15,19,40]$. For example, lymphocytic B-cell lymphoma is an extension of the primary diagnosis low-grade non-Hodgkin lymphoma. Experts of the field are asked for their opinion or for final confirmation/further details of primary diagnoses in relation to the diagnostic experience and available diagnostic techniques at the location of primary diagnosis.
Quality assurance refers to the minimization of diagnostic errors by discontinuous control of stains, diagnoses and by continuous education $[17,36]$. It is not directly involved in the diagnostic procedures; however, it has a long term influence on the daily practice [19]. These three different conditions reflect to the application of telepathology, which can be demonstrated as follows:

I. Primary diagnosis comprises the applied practices (a) frozen section service; (b) biopsy diagnosis; and (c) surgical specimen.

II. Secondary diagnosis is constituted of (a) expert consultations; (b) quantitative measurements; (c) biopsy diagnosis; (d) surgical specimen; and (e) autopsy (autopsy is subsummarized under this term as its clinical importance is usually not under time pressure)

III. Quality control includes (a) quantitative measurements; (b) continuous education and training; and (c) diagnostic inter-institutional trials.

The telepathology terms are related to the above mentioned compartments as follows:

The term active telepathology is used for interactive or remote microscope control and all functions are handled by the viewing pathologist. These systems have been developed in the last few years, especially for frozen section services or primary diagnosis. Secondary diagnosis can be performed with these systems as well; however, their primary application is primary diagnosis. Remote control telepathology systems provide the user with data banks which store all necessary information such as switch-on and switch-off time, viewed areas, chosen magnification, patients' identification and diagnosis [1,8,15]. Several systems from different providers are commercially available [19,31]. They are installed in an end-to-end system or masterclient arrangement. Systems from different companies are not compatible between each other, although efforts are now undertaken to create general telepathology standards with a common platform. In Japan, these efforts have finally resulted in a standard that allows systems from the companies Nikon, NTT, and Olympus to cooperate [22,23].

Passive telepathology is the management of transmitted images and information without interaction on the image acquisition side. These systems work in an off-line mode. The pathologist who requests additional diagnostic information acquires the images, stores the necessary patient's information in an adequate manner (for example, data bank), and transmits the com- 
plete information set to the expert. The selected expert works in a disconnected manner. He can chose date and time of response. The Internet is the most common transfer medium. It provides a common platform. Images can be transmitted as normal attachments to email, and no additional specific arrangements are necessary. This performance has been chosen for remote control measurements [6,30,31]. Of specific importance are static DNA measurements by use of the Internet and a specifically designed server (Euroquant) [6]. Several studies have shown the practicability of the Euroquant $[6,15]$. In addition to the high standard of the performed measurements the server guaranties a monitoring of performed Feulgen stains, image acquisition and transfer [6]. From this point of view, the Euroquant server is an integrated measurement/quality assurance system $[6,12,13,17]$.

Quality assurance systems have been designed mainly to train and educate students and young colleagues. Usually, they provide a fixed questionnaire with predefined answers compatible to multiple-choice questions. The more advanced systems select questions and answers in respect to the knowledge of the students, and provide the teachers with statistical data of the user, i.e., the progress of knowledge of the students [19, 36]. As a passive system they transfer data via Internet or intranet connections [4,39]. They are not equipped with self-learning executable programs at the moment to our knowledge.

\section{Technical compartments of telepathology systems}

Basically, telepathology systems require an image acquisition, data transfer and image display system. The necessary remote control of a microscope in case of primary diagnosis can be performed by commands transmitted to personnel working at the client microscope or by remote control of the client microscope. High standard cameras mounted on the microscope are a must in both primary and secondary diagnosis performance whereas cameras with lower pixel resolution can be used for additional acquisition of macroscopy images necessary for tissue block sampling [38,39,41, 42]. In (active) systems used for primary diagnosis, i.e., frozen section services the amount of image data (pixel $\times$ gray values) and the chosen transfer rate form a functional unit. The higher the image resolution to be transmitted the faster must be the transfer rate. Commonly, the ordinary TV standard (VHS) requires trans- fer rates no lower than 256 kbauds $(2 \times$ ISDN) in order to obtain a complete data transfer within a couple of seconds [10,32]. High resolution digital cameras which have a resolution of 3.3 megapixel or more need high speed data transfer usually only provided by glass fiber connection [2,15]. These data transfer lines are commonly available only in intranet connections within larger clinics or university grounds or only accessible on specific order $[10,33]$. In Europe, the most frequently used lines are those with ISDN standards. Trials with high speed wireless data transfer or with electrical power supply lines are promising, however, not generally available at the moment.

Passive telepathology systems have no direct connection between the image acquisition and image presentation in a functional manner. They commonly work via the Internet. Thus, both the sender and receiver work independent from each other, and the velocity of the line connections is of minor importance. It only influences the time needed for (automated) transmission to a storage system located at the receivers side. The recall speed of transmitted images can differ remarkably from the transmission speed. However, the transfer of images comprising more than 3-5 Mbytes can cause severe difficulties even under these conditions, and usually requires specific arrangements of the Internet provider. Passive expert consultation has the advantage the both the requesting and expert pathologists can work independently from each other, and have not to take into account the momentary work load of the partner $[9,13,34]$. On the other hand, they are missing the chance of interactive discussion of the case and contemporary selection of areas which might be of specific diagnostic importance [19]. According to the experiences published by the Armed Forces Institute of Pathology, these interactions seem not to be a prerequisite in telepathology expert consultations. A sufficient number of images (about 5-10 images/case), a detailed clinical history, and a clear cut diagnostic question permit in more than $90 \%$ of the requested consultations an accurate diagnostic statement [13]. That the Internet is an adequate transfer medium can be demonstrated in the exponential increase of electronic request for expert consultations: In 1999 about 650 requests have been applied to the Armed Forces Institute of Pathology, Washington; in the year 2000 more than 1200 consultations are expected (Internet address: www.afip.org/telepathology/).

A similar approach has been established at the Institute of Pathology, Charite Berlin sponsored by the UICC. Pathologists can ask for anonymous consulta- 
tion of cases which will be distributed to corresponding experts. The experts' opinion is retransmitted to the sender without mentioning the experts within a period of 48 hours.

Electronic image presentation is the limiting factor in all discussed telepathology applications. Commercially available cameras offer far better resolution than the TV screens in use. However, it can be expected that the technical progress will provide the user with screens offering a far advanced resolution within the near future, i.e., at time of public introduction of interactive TV systems [13].

\section{Present status of telepathology}

In Europe, the era of telepathology started with the first European Conference on Telepathology held in Heidelberg, June 1992 [9,10]. Although first efforts can be followed back to 1970 when the first interactive telepathology system was installed between the Logan airport in Boston and the Institute of Pathology at the Massachusetts General Hospital, the year 1992 can be considered as milestone. From Tromsoe in Norway, promising reports of routine interactive telepathology and from Heidelberg routine passive telepathology sessions have been published at this time [14,24]. Nowadays, several telepathology systems are commercially available (for example, Histkom, Leica, Nikon, NTT, Olympus, Zeiss). They are primarily designed for interactive telepathology, and several of them are in routine use. Larger Institutes of Pathology provide small hospitals located in their neighborhood with frozen section services, in Germany, for example, in Berlin, Dresden, Giessen and Tübingen. A private Institute of Pathology located in Aurich, Northern Germany uses a non-remote control telepathology system with oral commands for frozen section services, and reports good results [35]. In summary, the application of telepathology for primary diagnosis has left its childhood at the end of the last century.

Telepathology use for expert consultation or secondary diagnosis has made a great progress with the increased distribution of Internet access and e-mail transfer. The largest expert consultation institution in pathology, the Armed Forces Institute of Pathology, Washington reports an exponentially increase of telepathology expert consultations in their institution within the last years. This institution started its passive teleconsultation service 1995, and only a couple of cases were electronically submitted in 1995 and 1996.
Nowadays, this number has reached the number of about 1200 telepathology consultations per year. Our own experiences point in the same direction: the frequency of requested expert consultations amounts to about 3-6 cases/week, and has still an increasing tendency.

In future, telepathology system will probably spread into smaller institutions, and into international collaboration [13,19]. Active telepathology systems are needed in countries with only small number of pathologists, and will be installed in a central institution and hospitals located in their neighborhood. They will probably form independent networks. Interestingly, in Japan, telepathology networks have been created in five districts which each connect about 3-7 telepathology systems of different companies [22,23]. These networks work on their own basis and have no connections in between. They provide small hospitals with frozen section services, and, in addition, the possibility of expert consultations between departments of pathology located in the corresponding prefecture [22]. In general, however, passive telepathology systems are more adequate for expert consultation, and allow the consultation of institutions located in far distances or foreign continents. In respect to the existing time gap between different countries or continents, for example, between Asia and the USA, this unavoidable constraint can also be used in advantage for several purposes: The main handicap of some institutes of pathology is the basic order of workflow, namely tissue performance, diagnostic statements of the involved pathologists, the dictation of the findings, and the typing performed by secretaries. Often, the working hours of secretaries do not allow a typing of records at the same day, which has then to be postphoned. When transmitting the dictated findings to an institution located in a continent with a sufficient time gap the records can be typed within the local working hours, and the typed diagnoses can be retransmitted at night time. In such a manner, the local institute of pathology can save time for final diagnosis, and serve the patients in a more appropriate way [44]. The described performance of secretary work is already a daily practice in some institutes of the USA which submit their dictates to institutes located in India [44]. There is no doubt that this so-called globalization in telepathology can also be used for other purposes, for example, to save time in the diagnostic work or in the set up of request for additional technical procedures in connection with the primary diagnosis. Trials to analyze the performance and diagnostic accuracy of the so-called globalization are ongoing. The legal 
aspects are not yet clarified; however, in Europe no major problems should be expected (see also C. Dierks "Legal aspects of telepathology", in this issue).

In conclusion, telepathology is not only a subsidy of the lack of pathologists in certain hospitals; it is, in contrast, an extension of diagnostic pathology and will probably become great influence in the daily work and practice. For sure, it has left its childhood and is on the way to broad applications which will involve pathology, related medical disciplines, social aspects and the medical progress.

\section{References}

[1] C. Busch and S. Olsson, Future strategy for telepathology trial in Sweden: higher resolution, real time transmission in a multipurpose work station for diagnostic pathology, Arch. Anat. Cytol. Pathol. 43 (1995), 242-245.

[2] D. Daniel, J. Schlesner, K. Wehrstedt, P. Hufnagl and M. Dietel, Influence of image resolution on image quality in telepathology, Elec. J. Pathol. Histol. 6(3) (2000), No. 003-08.

[3] T.J. Eide, I. Nordrum and H. Stalsberg, The validity of frozen section diagnosis based on video-microscopy, Zentralbl. Pathol. 138 (1992), 381-436.

[4] P. Gombas, Can static telepathology be a trusted method for the communication of pathological information? The image pyramid, Elec. J. Pathol. Histol. 6(3) (2000), No. 003-04.

[5] L. Goncalves and C. Cunha, Telepathology experiences in Portugal, Elec. J. Pathol. Histol. 2(4) (1995), 954-959.

[6] G. Haroske, A. Boecking, W. Meyer, K. Kayser, D. Kunze and M. Oberholzer, EUROQUANT - a quantitation server for remote DNA image cytometry, Elec. J. Pathol. Histol. 4(4) (1997), No. 974-08.

[7] B.A. Harris, Jr., Telemedicine: a glance into the future (editorial; comment), Mayo Clin. Proc. 69 (1994), 1212.

[8] P. Hufnagl and T.N. Nguyen-Dobinsky, Telepathology using TPS, Elec. J. Pathol. Histol. 6(3) (2000), No. 003-10.

[9] K. Kayser, M. Drlicek and W. Rahn, Aids of telepathology in intraoperative histomorphological tumor diagnosis and classification, In vivo 7 (1993), 379-382.

[10] K. Kayser and M. Drlicek, Visual telecommunication for expert consultation of intraoperative sections, Zentralbl. Pathol. 138 (1992), 381-436.

[11] K. Kayser, P. Fritz and M. Drlicek, Aspects of telepathology in routinary diagnostic work with specific emphasis on ISDN, Arch. Anat. Cytol. Pathol. 43 (1995), 216-218.

[12] K. Kayser and G. Kayser, Telepathology - aspects of social influence and quality control, Elec. J. Pathol. Histol. 3(3) (1996), No. 963-04.

[13] K. Kayser, G. Kayser and S. Zink, New technical aspects in telepathology, Elec. J. Pathol. Histol. 6(3) (2000), No. 003-04.

[14] K. Kayser, M. Oberholzer, G. Weisse, I. Weisse and H. von Eberstein, Long distance image transfer: First results for its use in histopathological diagnosis, Acta Pathol. Microbiol. Immunol. Scand. 99 (1991), 808-814.
[15] K. Kayser, J. Szymas and R. Weinstein, Telepathology Telecommunication, Electronic Education and Publication in Pathology, Springer Heidelberg, New York, 1999.

[16] K. Kayser, G. Weisse, H. von Eberstein, I. Weisse and H. Frank, Histomorphologische Diagnostik am Telefon - Vision oder Realitaet, Labor Praxis (Nov. 1989), 1020-1024.

[17] K. Kayser and G. Kayser, Recent development of telepathology in Europe with specific emphasis on quality assurance, J. Anal. Quant. Cytol. Histol. 21 (1999), 319-328.

[18] K. Kayser, Telemedizin, Wiener Klin. Wschr. 108 (1996), 932940.

[19] K. Kayser, Telepathology in Europe. Its practical use, Arch. Anat. Cytol. Pathol. 43 (1995), 196-199.

[20] L.P. Kok, R. van Drunen, M.E. Boon, S. Beck and R.S. Coevorden, General practitioners use digital cameras and Internet for telepathology of skin lesions, Elec. J. Pathol. Histol. 6(3) (2000), No. 003-01.

[21] E. Martin, P. Dusserre, C.L. Got, A. Vieillefond, B. Franc, G. Brugal and B. Retailliau, Telepathology in France. Justifications and developments, Arch. Anat. Cytol. Pathol. 43 (1995), 191-195.

[22] T. Mazaki, Y. Enoki and Y. Tsuchihashi, Telepathology network in Kyoto, Japan. Pathol. Intern. 50 (2000), A206.

[23] H. Nakamine, E. Nishino, K. Ono, H. Akamatu and T. Sanke, Practical use of telepathology for intraoperative pathology diagnosis, establishment of a telepathology network, Pathol. Intern. 50 (2000), A206.

[24] I. Nordum, B. Engum, E. Rinde, A. Finseth, H. Ericsson, M. Kearney, H. Stalsberg and T.J. Eide, Remote frozen section service: a telepathology project in northern Norway, Hum. Pathol. 22 (1991), 514-518.

[25] B. Nymo and B. Engum, Telemedicine to improve the quality, availability and effectiveness of the health service in rural regions, Kjeller, TF-lecture F10/90, Norwegian Telecom Research (1990).

[26] P. Oberbarnscheidt, P. Hufnagl, H. Guski, S. Hauptmann and M. Dietel, Analysis of errors in telepathology, Elec. J. Pathol. Histol. 6(3) (2000), No. 003-11.

[27] M. Oberholzer, H.R. Fischer, H. Christen, S. Gerber, M. Bruehlmann, M. Famos, C.H. Winkler, P. Fehr and L. Baechtold, Telepathology with an Integrated Services Digital Network: a new tool in pathology, in: Compendium on the Computerized Cytology and Histology Laboratory, G.L. Wied, P. Bartels, D. Rosenthal and U. Schenck, eds, Tutorials of Cytology, Chicago, 1994, pp. 295-305.

[28] M. Oberholzer, H.R. Fischer, H. Christen, S. Gerber, M. Bruehlmann, M. Mihatsch, M. Famos, C. Winkler, P. Fehr, L. Baechtold and K. Kayser, Telepathology with ISDN - A new tool for image transfer in surgical pathology, Hum. Pathol. 24 (1993), 1078-1085.

[29] M. Oberholzer, H.R. Fischer, H. Christen, S. Gerber, M. Bruehlmann, M.J. Mihatsch, T. Gahm, M. Famos, C. Winkler, P. Fehr, H.J. Hosch and L. Baechtold, Telepathology: frozen section diagnosis at a distance, Virchows Arch. 426 (1995), 3-9.

[30] S. Olsson and C.H. Busch, A national telepathology in Sweden. Feasibility and assessment, Arch. Anat. Cytol. Pathol. 43 (1995), 234-241. 
[31] P. Schwarzmann, J. Schmid, C.I. Schnoerr, G. Straessle and S. Witte, Telemicroscopy stations for telepathology based on broadband and ISDN connections, Arch. Anat. Cytol. Pathol. 43 (1995), 209-215.

[32] P. Schwarzmann, Telemicroscopy. Design considerations for a key tool in telepathology, Zentralbl. Pathol. 138 (1992), 381436.

[33] S. Seiwerth, Telepathology and teleradiology, Elec. J. Pathol. Histol. 6(3) (2000), No. 003-06.

[34] J. Slodkowska, K. Kayser, P. Radomski and P. Papierz, First experiences in a telepathology consultation service, Elec. J. Pathol. Histol. 6(3) (2000), No. 003-07.

[35] G. Stauch, K.W. Schweppe and M. Puetz, One year experience with telepathology for frozen sections, Elec. J. Pathol. Histol. 2(4) (1995), 954-958.

[36] J. Szymas, Telepathology in Poland, present status and future aspects, Elec. J. Pathol. Histol. 6(3) (2000), No. 003-02.

[37] J. Szymas, G. Wolf, W. Papierz, M. Danilewicz and B. Jarosz, Feasibility of telepathology in neurooncological diagnosis: a prospective study allowing the analysis of histological images and cases to be viewed by several partners, Elec. J. Pathol. Histol. 6(3) (2000), No. 003-05.

[38] A. Vieillefond, F. Staroz, M. Fabre, P. Bedossa, V. Martin-Pop, E. Martin, G. Got and B. Franc, Reliability of the anatomo- pathological diagnosis by static image transfer, Arch. Anat. Cytol. Pathol. 43 (1995), 246-250.

[39] K. Wehrstedt, C. Tennstedt and P. Hufnagl, Telepathology in fetal pathology, Elec. J. Pathol. Histol. 6(3) (2000), No. 00309.

[40] R.S. Weinstein, Prospects for telepathology, Human Pathol. 17 (1986), 433-434.

[41] R.S. Weinstein, A. Bhattacharyya, Y.P. Yu, J.R. Davis, J.M. Byers, A.R. Graham and R. Martinez, Pathology consultation services via the Arizona-International telemedicine network, Arch. Anat. Cytol. Pathol. 43 (1995), 219-226.

[42] R.S. Weinstein, K.J. Bloom, E.A. Krupinski and L.S. Rozek, Human performance studies of the video microscopy component of a dynamic telepathology system, Zentralbl. Pathol. 138 (1992), 381-436.

[43] R.S. Weinstein, K.J. Bloom and R.S. Rozek, Static and dynamic imaging in pathology, in: Image Management and Communications in Patient Care: Implementation and Impac, S.K. Mun, M. Greberman, W.R. Hendee, R. Shannon, eds, IEEE Computer Soc. Press, Los Alamitos, CA, 1990, pp. 77-85.

[44] R.S. Weinstein, Personnel communication, 2000. 


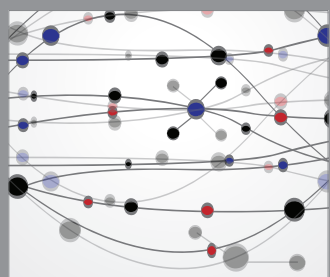

The Scientific World Journal
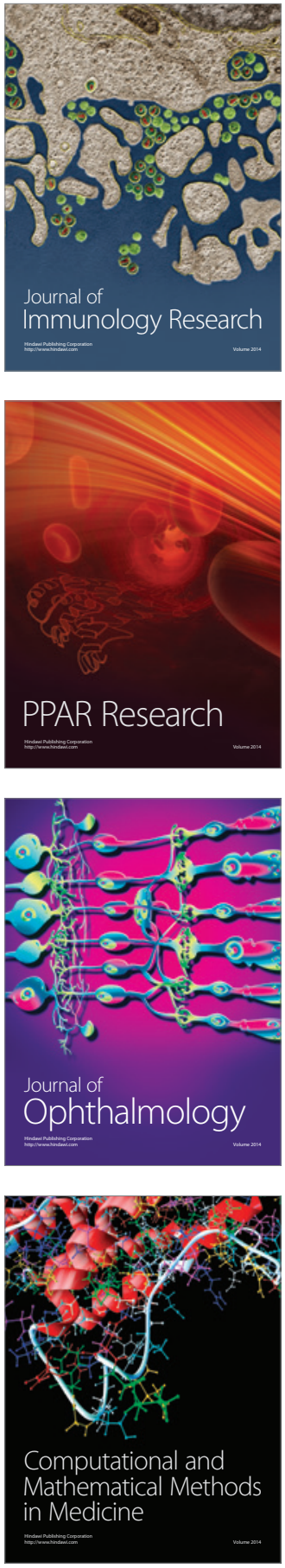

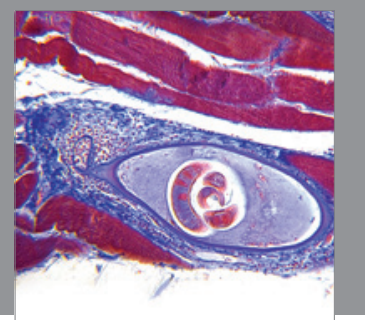

Gastroenterology

Research and Practice
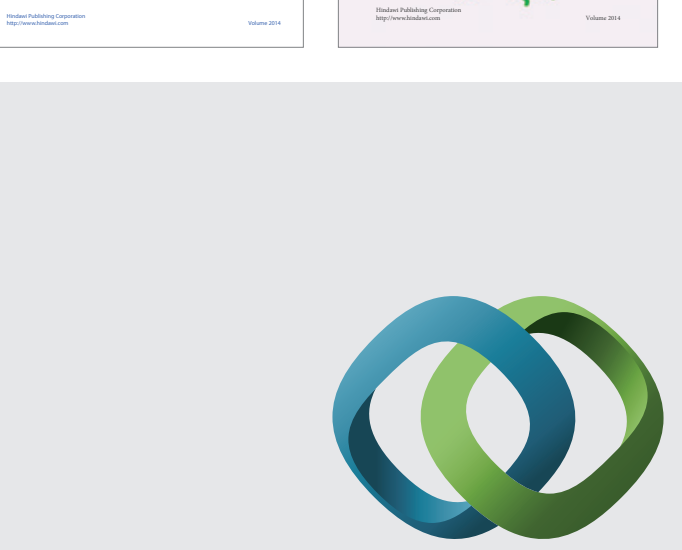

\section{Hindawi}

Submit your manuscripts at

http://www.hindawi.com
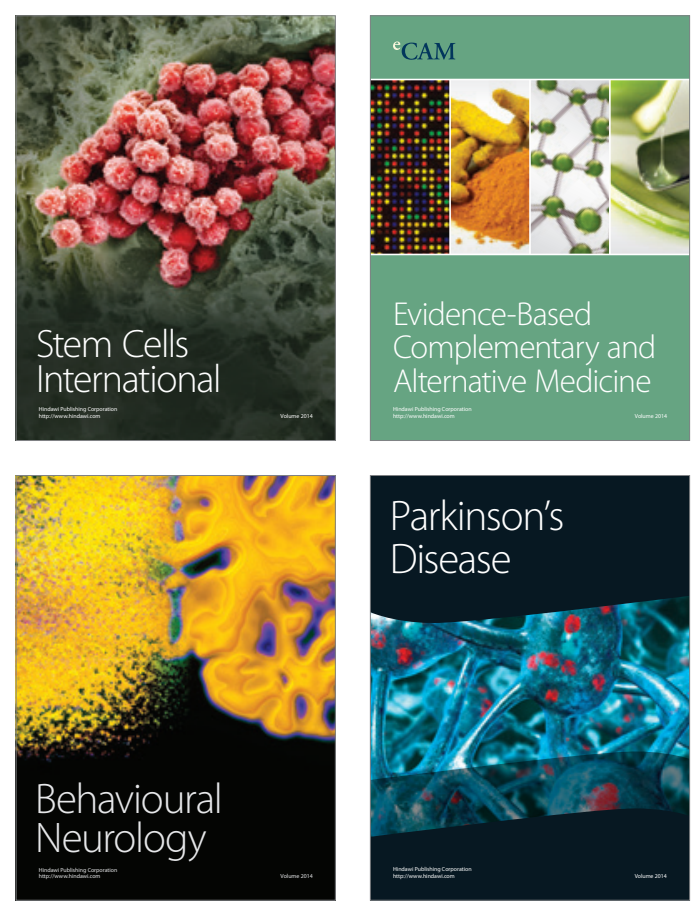

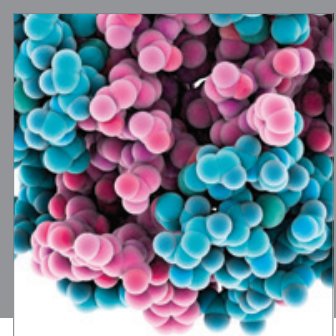

Journal of
Diabetes Research

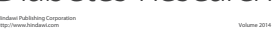

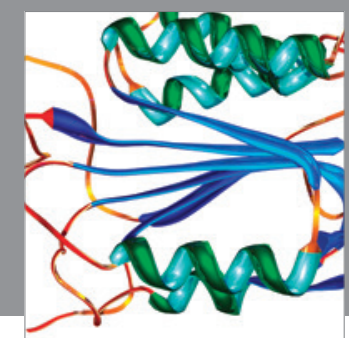

Disease Markers
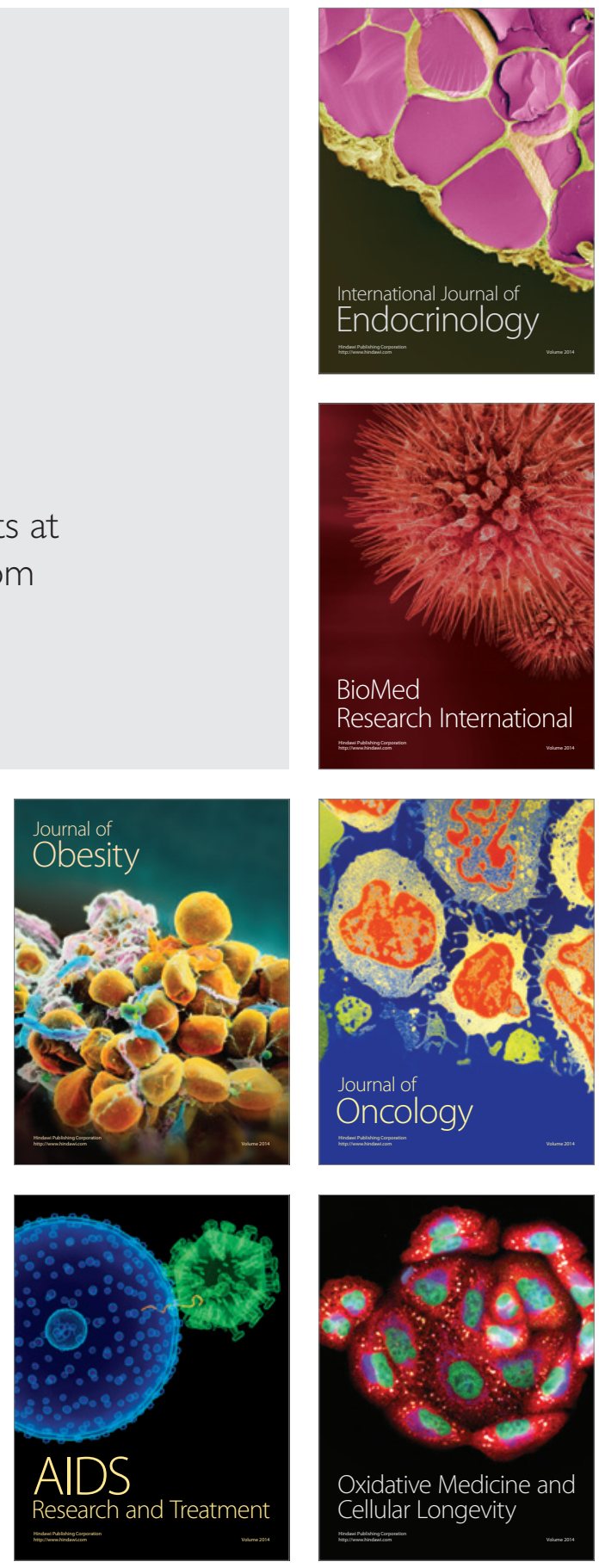